\title{
Pigment-induced Crystallization in Colored Plastics Based on Partially Crystalline Polymers
}

\author{
Shigeru Suzuki and Jin Mizuguchi* \\ Toyo Ink Manufacturing Co., Ltd. \\ 104-8377 Tokyo, Japan \\ * Department of Applied Physics, Yokohama National University \\ 240-8501 Yokohama, Japan
}

\begin{abstract}
Mold shrinkage of the colored plastics based on partially crystalline polymers is a well-known phenomenon and is assumed to be caused by pigment-induced crystallization of polymers. A systematic of investigation has been carried out for a variety of organic pigments in polypropylene (PP) and polyethylene (HDPE) systems in order to clarify the correlation between the nucleation effect due to pigments and the mold shrinkage. Special attention was paid to the onset temperature of crystallization for both PP and HDPE systems as monitored by DSC (differential scanning colorimeter). In parallel, the crystallization rate was also studied in the isothermal process by depolarization method for PP while by dilatometry for HDPE systems. As a result, the mold shrinkage is found to become more significant as the onset temperature is higher and the crystallization rate is faster. This tendency is well correlated with the formation of small spherulites (diameter: less than $1 \mu \mathrm{m}$ ). These results lead us to conclude that the organic pigments in colored plastics serve as a nucleation agent for partially crystalline polymers to form small spherulites, causing the mold shrinkage to occur.
\end{abstract}

Keywords: organic pigment, mold shrinkage, polymer crystallization, nucleation, polyolefin 


\section{INTRODUCTION}

In the field of colored plastics, especially in poly-olefins such as polypropylene (PP) and high density polyethylene (HDPE), organic pigments are known to remarkably affect the mold shrinkage or distortion [1-3]. Turnbull-Fischer [4] and Lauritzen-Hoffman [5] pointed out that the pigment particles dispersed as colorants initiate crystallization of partially crystalline polymers. In addition, a number of reports were also published on the nucleating effect in polymers due to pigments [6-12]. However, these are the studies based on the approach from the research area of polymers, not from pigments. For this reason, an attempt was made in the present investigation to clarify the influence of organic pigments on the crystallization of polymers and eventually the mold shrinkage in PP and HDPE systems. We believe that the present information could provide us with a guiding principle how to modify pigment surface or tailor pigments themselves.

Special attention has been paid in the present investigation to the onset temperature of crystallization in both pigment-loaded PP and HDPE systems as monitored by DSC (differential scanning colorimeter). In parallel, the crystallization rate was also studied in the isothermal process (at a fixed temperature) by depolarization method for PP while by dilatometry for HDPE systems. The former method is simple but still sufficient for slow crystallization processes as in the case of PP systems. However, this method is obviously insufficient for HDPE systems in which the crystallization proceeds quite rapidly; whereas dilatometry is adequate for this purpose.

\section{EXPERIMENTAL}

\section{Materials and experimental conditions}

Tables 1 and 2 detail the commercial PP and HDPE, as well as organic and inorganic pigments used in the present experiment, respectively. The composition of the colored plastics is given in Table 3. The molding conditions are summarized in Table 4. The test plaque used for the injection-mold test has a dimension of $120 \times 80 \times 2 \mathrm{~mm}^{3}$.

\section{Measurements:}

\section{Mold shrinkage}

The extent of mold shrinkage was measured for two directions: one is along the machine 
direction (MD) and the other is perpendicular to the machine direction (TD: transverse direction).

\section{Onset temperature of the crystallization $(T p)$}

The onset temperature (Tp) of the crystallization was measured by DSC for both PP and HDPE systems. The test samples were heated, respectively, to 230 and $180{ }^{\circ} \mathrm{C}$ for PP and HDPE systems and then cooled at a rate of $5{ }^{\circ} \mathrm{C} / \mathrm{min}$. The exothermal peak upon crystallization gave the onset temperature.

Crystallization rate measured in the isothermal process for pigment-loaded PP and HDPE systems

The crystallization rate was measured in the isothermal process at fixed temperatures of 130 and $129{ }^{\circ} \mathrm{C}$ for PP and HDPE systems, respectively. The half time $t_{1 / 2}$ is defined as the time required for $50 \%$ crystallization. The following two different methods were employed for measurements

The depolarization method, applied for PP systems, utilizes a pair of polarizers which are arranged in a cross-nicol position: no light transmission (dark). Then, some molten samples of colored plastics are placed between these polarizers. Light transmission occurs in proportional to the extent of the crystallization with elapsed time. The intensity of transmitted light is used as the measure for the degree of crystallization.

Dilatometry, applied for HDPE systems, is a method to measure the change in volume caused by the crystallization of polymers, using a glass capillary together with mercury [13]. This method enables us to carry out a very precise measurement even for rapidly changing processes in a close temperature range.

\section{Microscope observation}

A polarizing microscope was used to observe spherulites of polymers which appear as a result of the crystallization. The size of the spherulite serves as a good measure of the crystalline state. When the nucleation frequency is high, we normally observe small spherulites. On the contrary, the spherulites grow up to large crystallites as the nucleation frequency decreases.

\section{RESULTS AND DISCUSSION}

\section{Effect of pigments on the mold shrinkage in PP and HDPE systems}


Figs. 1 and 2 show the MD/TD mold shrinkage in PP and HDPE systems loaded with a variety of pigments, respectively. In Fig.1 for PP systems, the blank sample without any pigment finds itself at the lower-left corner in zone 1. MD shrinkage in PP systems is rather insignificant in all pigments. On the other hand, the organic pigments in zone 3 exhibit considerable TD shrinkage as compared with the blank sample in zone 1 . The inorganic pigments in zone $1\left(\mathrm{TiO}_{2}, \mathrm{Fe}_{2} \mathrm{O}_{3} \& \mathrm{Cd} \mathrm{Y}\right)$ are quite inactive for the mold shrinkage.

In HDPE systems, the blank sample is present at the upper-left corner in zone 1. It is also to be noted that the organic pigments in zone 3 exhibit quite significant MD shrinkage but less TD shrinkage. The MD shrinkage causes appreciable distortion in molded products in practice. The inorganic pigments in zone 1 behave again nearly in the same way as the blank sample.

\section{Correlation between the onset temperature of crystallization (Tp) and the mold shrinkage in PP and HDPE systems}

Figs.3 and 4 show the correlation between the onset temperature of crystallization [Tp] and the mold shrinkage in PP and HDPE systems, respectively. In both systems, a good linearity is recognized between the mold shrinkage and the onset temperature. The inorganic pigments in both systems exhibit insignificant mold shrinkage. It is also remarkable to note that the mold shrinkage of GR (condensed azo: CIBA) in HDPE systems is smaller as compared with that of the blank sample.

\section{Correlation between the crystallization rate and the mold shrinkage in PP and HDPE systems}

Figs. 5 shows the correlation between the time required for $50 \%$ crystallization $\left(t_{1 / 2}\right)$ and the mold shrinkage in PP systems. The half time was measured in the isothermal process at a fixed temperature of $130{ }^{\circ} \mathrm{C}$ by means of depolarization method. Fig.6 shows the same correlation for HDPE systems in which the measurements were made at $129^{\circ} \mathrm{C}$ by means of dilatometry. Both PP and HDPE systems exhibit the same tendency that the decreased mold shrinkage is in line with increasing $t_{1 / 2}$, i.e. slow crystallization rate. In PP systems, $\mathrm{Fe}_{2} \mathrm{O}_{3}$ is found to be exceptionally inactive for the mold shrinkage.

Fig.7 shows the three representative examples (1090, GR and 2GLT) of the detailed 
crystallization process as a function of time. The volume fraction of the crystallized phase $(X)$ is obtained by dilatomerty measurements. The volume fraction of the non-crystallized phase (1-X ) (i.e. extent or degree of crystallization) is then calculated and given in the present figure. It is to be noted that 2GLT (isoindolinone) shows an abrupt crystallization around $280 \mathrm{~min}$. This phenomenon corresponds to a supercooling effect as found in water. The same effect was also observed in 3RLT (again isoindolinone) but not shown here. It is, however, not yet fully clarified whether the supercooling effect here is specific of isoindolinone pigments.

\section{Correlation between the spherulite size and the mold shrinkage in PP systems}

Table 5 shows the spherulite size in PP systems as observed by a polarizing microscope, together with the values for the onset temperature of crystallization (Fig.3) as well as the time required for $50 \%$ crystallization $\left(t_{1 / 2}\right)$ (Fig.5). The "increase in Tp" given in the table is measured relative to the onset temperature of the blank sample. Judging from the present table, the mold shrinkage as characterized by the increase in Tp (Fig.3) and the fast crystallization rate (Fig.5) becomes more significant as the spherulite size is smaller. The formation of small spherulites is equivalent to saying that the nucleation frequency is high in PP systems. Unfortunately, the corresponding results in HDPE systems are not available because their spherulites are generally too small to observe by microscope. However, the same situation as that in PP systems is presumably also the case in HDPE systems

It is also important to note that the inorganic pigments in Table 5 (which are inactive for the mold shrinkage) exhibit some increase in $T p$ in spite of slow crystallization rate and large spherulites. This would suggest that the spherulite size plays the most important role in the occurrence of mold shrinkage that exceeds other influencing factors such as high onset temperature and slow crystallization rate. In conclusion, the mold shrinkage becomes more significant as the nucleation frequency is increased in colored plastic systems.

\section{Mechanism of the mold shrinkage}

On the basis of the forgoing discussions, the mechanism of mold shrinkage can be explained in the following way. The presence of organic pigments in colored plastics 
enhances the nucleation frequency in partially crystalline polymers. This, in turn, induces a change in higher order structure of polymer systems. Then, the internal stress may remain in bulk or be propagated throughout the system during the cooling process, thus leading to the formation of a residual stress in polymers. This eventually brings about mold shrinkage in colored plastics.

\section{CONCLUSIONS}

Organic pigments are found to enhance the nucleation frequency of polymers that induces internal stress, i.e. a change in higher order structure of polymers. This may eventually lead to a residual stress which results in mold shrinkage of colored plastics. The present conclusion strongly suggests that the surface treatment and/or surface modification of pigments play a decisive role for the suppression of mold shrinkage in colored plastics.

\section{ACKNOWDEDGEMENTS}

The authors appreciate Toyo Ink Manufacturing Co., Ltd. for the approval of the present publication. The authors are also indebted to Messrs. H. Maki and S. Miyashita for suggestions and discussion. 


\section{REFERENCES}

1. Herbst, M. and Hunger, K., Industrial Organic Chemistry, VCH Weinheim, 1993, p.73.

2. Suzuki, S., Dyestuffs \& Chemicals, 1977, 22, 69 (in Japanese).

3. Ando, G. and Suzuki, S., Industrial Materials, 1977, 25, 29 (in Japanese).

4. Turnbull, D. and Fischer, J.C., J. Chem. Phys., 1949, 17, 71.

5. Lauritzen, J. I., Jr. and Hofman, J.D., J. Res. Nat. Bur. Stds., 1961, 65A, 257.

6. Silberman, A., Raninson, E., Dolgopolsky, I. and Kenig, S., Polym. Adv. Technol., 1995, 6, 643.

7. Jacoby, P., Bersted, B. H., Kissel, W. J. and Smith, C. E., J. Polym. Sci., Part B: Polym. Phys., 1986, 24, 461.

8. Filho, D. S. and Oliveira, C. M. F., Makromol. Chem., 1993, 194, 285.

9. Sterzynski, T., Calo, P., Lambla, M. and Thomas, M., Polym. Eng. Sci., 1997, 37, 1917.

10. Menges, G., Sarholz, R. and Kruger, E., Plastverarbeiter, 1981, 32, 59.

11. Włochowicz, A. and Eder, M., Angew. Macromol. Chem., 1989, 171, 79.

12. Turturro, A., Olivero, L., Pedemonte, E. and Alfonso, G. C., Br. Polym. J., 1973, 5, 129.

13. Suzuki, S., Miyashita, S. and Katabuchi, H., J. Japan Soc. Colour Mat., 2001, 74, 379 (in Japanese). 
Figure captions

Fig.1 MD and TD mold shrinkages in PP systems for a variety of pigments. Molding temperature: $220^{\circ} \mathrm{C}$. "Blank" denotes the plastic sample without any pigment while "DA" is the sample which includes only a dispersing agent (see footnote of Table $3)$.

Fig.2 MD and TD mold shrinkages in PP systems for a variety of pigments. Molding temperature: $240^{\circ} \mathrm{C}$. "Blank" denotes the plastic sample without any pigment while "DA" is the sample which includes only a dispersing agent (see footnote of Table $3)$.

Fig.3 TD shrinkage versus onset temperature of crystallization $\left(T_{P}\right)$ in pigment-loaded PP systems.

Fig.4 MD shrinkage versus onset temperature of crystallization $\left(T_{P}\right)$ in pigment-loaded HDPE systems.

Fig.5 TD shrinkage versus half time of crystallization in pigment-loaded PP systems measured in the isothermal process $\left(130^{\circ} \mathrm{C}\right)$ by means of depolarization method.

Fig.6 MD shrinkage versus half time of crystallization in pigment-loaded HDPE systems measured in the isothermal process $\left(129^{\circ} \mathrm{C}\right)$ by means of dilatometry.

Fig.7 The volume fraction of the non-crystallized phase (i.e. degree of crystallization) is plotted as a function of time in the isothermal process of $129{ }^{\circ} \mathrm{C}$. The volume fraction of the crystallized phase $(\chi)$ is determined by experiment [13]. Then, the volume fraction of the non-crystallized phase $(1-\chi)$ is deduced as given in the present figure. 
Table 1 Polyolefins used

\begin{tabular}{lcccc}
\hline Polymer & Trade name & Manufacturer & MFR* & Type \\
\hline HDPE & Hi-zex 2208 J & Mitsui Chemical & 8 & High density polyethylene \\
PP & Mitsubishi Noblen MH-4 & Mitsubishi Chemical & 5 & Isotactic homopolymer \\
\hline *Melt flow rate $(\mathrm{g} / 10 \mathrm{~min})$
\end{tabular}

Table 2 Pigments used

\begin{tabular}{|c|c|c|c|c|}
\hline Trade name & Manufacturer & Pigment class & C.I.name & Abbreviation \\
\hline Iragazin Yellow 2GLT & Ciba & Isoindorinone & P.Y.109 & $2 \mathrm{GLT}$ \\
\hline Iragazin Yellow 3RLT & Ciba & Isoindorinone & P.Y.110 & 3RLT \\
\hline Cromophtal Yellow GR & Ciba & Condensed azo & P.Y.95 & GR \\
\hline Lithol Fast Yellow 1090 & BASF & Quinophthalone & P.Y.138 & 1090 \\
\hline Cinquasia Red Y & Ciba & Quinacridone & P.V.19 & Red Y \\
\hline Cromophtal Scarlet R & Ciba & Condensed azo & P.R.166 & Sc $\mathrm{R}$ \\
\hline Lionol Green 2YS & Toyo Ink & Cyanine green & P.G.7 & $2 Y S$ \\
\hline Lionol Green GLS * & Toyo Ink & Cyanine green & P.G.7 & GLS \\
\hline Heliogen Blue 6911K & BASF & Cyanine blue & P.B.15:3 & $6911 \mathrm{~K}$ \\
\hline Lionol Blue BLS* & Toyo Ink & Cyanine blue & P.B.15:1 & BLS \\
\hline TE Yellow & Toyo Ink & Mixture** & - & TE \\
\hline Tipaque CR-50 & Ishihara & $\mathrm{TiO}_{2}$ & P.W.6 & $\mathrm{TiO}_{2}$ \\
\hline Cd Yellow 2240 & Orient Chem. & Cd yellow & P.Y.37 & $\mathrm{CdY}$ \\
\hline Toda Color 120ED & Toda & Iron oxide & P.R.101 & $\mathrm{Fe}_{2} \mathrm{O}_{3}$ \\
\hline Mitsubishi Carbon \#45 & Mitsubishi Chem. & Carbon black & P.BL.7 & $\mathrm{CB}$ \\
\hline
\end{tabular}

*Shrinkage-improved product

**Shrinkage-improved product by surface coating and its composition::

2GLT/3RLT/TiO2/Zn-stearate $=17 / 6 / 60 / 17$ (in weight)

Table 3 Composition of colored plastics

\begin{tabular}{ccc}
\hline Pigment type & Weight ratio of pigment/DA*/polymer & Measurements \\
\hline Org. \& inorg. Pigments & $0.2 / 0.07 / 100$ & \multirow{2}{*}{ Dilatometry } \\
TE & $0.65 / 0 / 100$ & \\
\hline Organic pigments & $0.1 / 0.1 / 100$ & \multirow{2}{*}{ Mold shrinkage \& DSC } \\
Inorganic pigments & $0.2 / 0.1 / 100$ & \\
TE & $0.65 / 0 / 100$ & \\
\hline * Dispersing agent: a mixture of $\mathrm{Zn}$ and Ca stearates for PP and Zn-stearate for HDPE
\end{tabular}

Table 4 Molding conditions

\begin{tabular}{cccccccccc}
\hline \multirow{2}{*}{ Polymer } & \multicolumn{2}{c}{ Temperature $\left({ }^{\circ} \mathrm{C}\right)$} & & \multicolumn{2}{c}{ Pressure $\left(\mathrm{kg} / \mathrm{cm}^{2}\right)$} & & \multicolumn{2}{c}{ Time for molding process $(\mathrm{s})$} \\
\cline { 2 - 3 } & Cylinder & Mold & & Injection & Hold-on & & injection & Hold-on & Cooling \\
\hline HDPE & 240 & 40 & & 80 & 40 & & 5 & 3 & 15 \\
PP & 220 & 40 & & 55 & 40 & & 4 & 2 & 14 \\
\hline
\end{tabular}


Table 5 Parameters for the crystallization process of PP systems

\begin{tabular}{cccc}
\hline Pigment & Increase in $T p\left({ }^{\circ} \mathrm{C}\right)$ & $t_{1 / 2}(\mathrm{~min})$ & Spherulite size* \\
\hline Blank & 0 & 18.8 & large \\
$2 \mathrm{GLT}$ & 0 & 3 & small \\
$\mathrm{GR}$ & 4 & 23.5 & large \\
$\mathrm{RedY}$ & 15 & 1.6 & small \\
$\mathrm{ScR}$ & 8 & 6.3 & small \\
$2 \mathrm{YS}$ & 11 & 2.6 & small \\
$\mathrm{GLS}$ & 10 & 3.6 & medium \\
$6911 \mathrm{~K}$ & 13 & 1.4 & small \\
$\mathrm{BLS}$ & 6 & 8.9 & medium \\
\hline $\mathrm{TiO}_{2}$ & 3 & 16.5 & large \\
$\mathrm{Cd}^{\mathrm{H}}$ & 7 & 13.1 & large \\
$\mathrm{Fe}_{2} \mathrm{O}_{3}$ & 7 & 4.1 & large \\
\hline $\mathrm{CB}$ & 3 & 13.6 & large
\end{tabular}

*"large"; Several $10 \mu \mathrm{m}$, "medium"; one to several $10 \mu \mathrm{m}$, "small"; less than $1 \mu \mathrm{m}$.

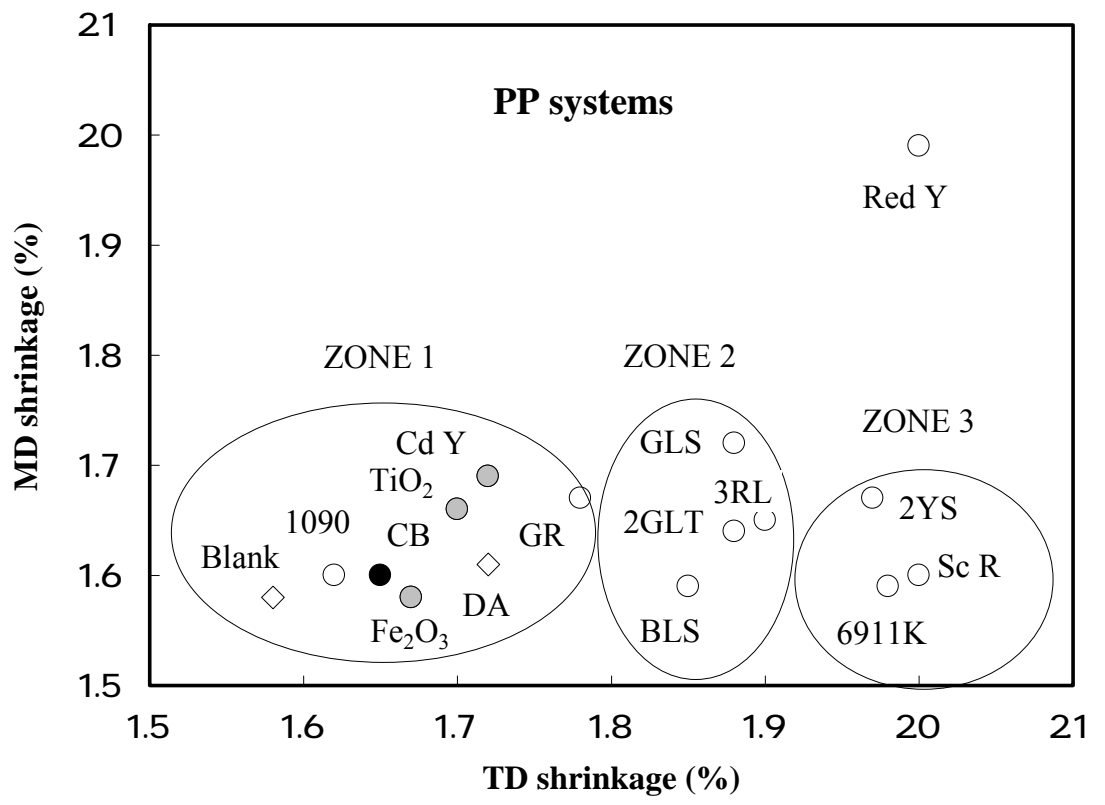

Fig. 1

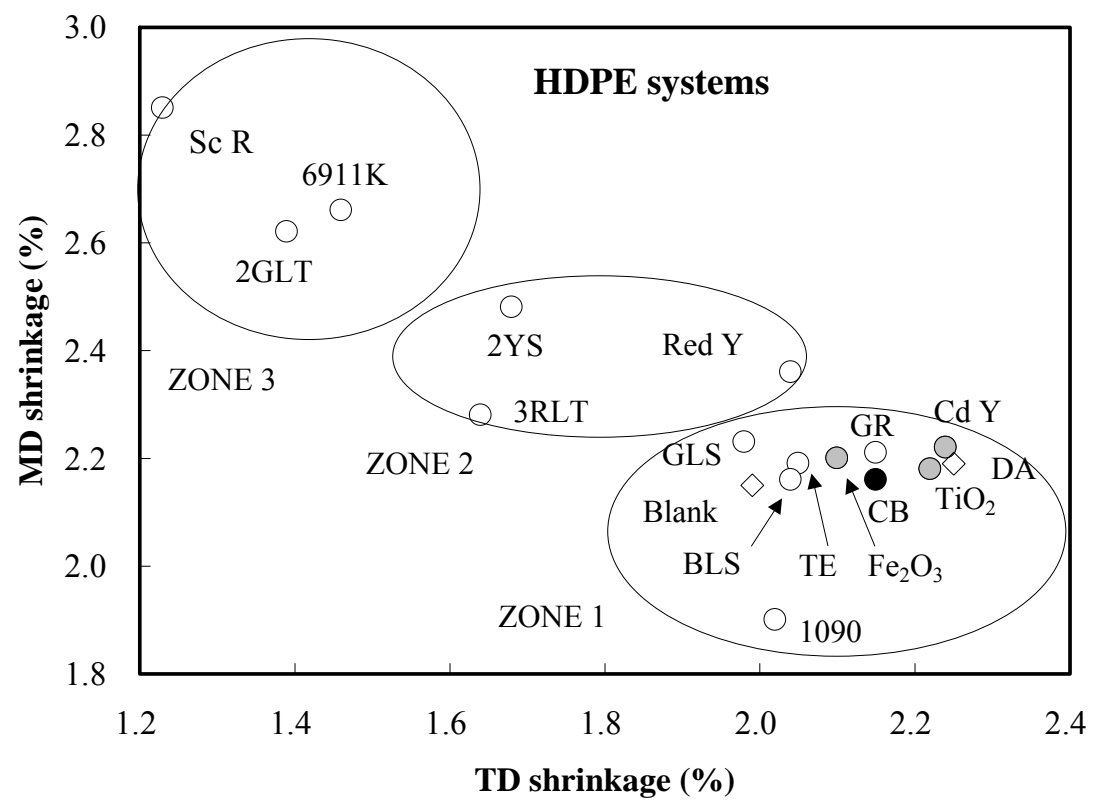

Fig. 2 


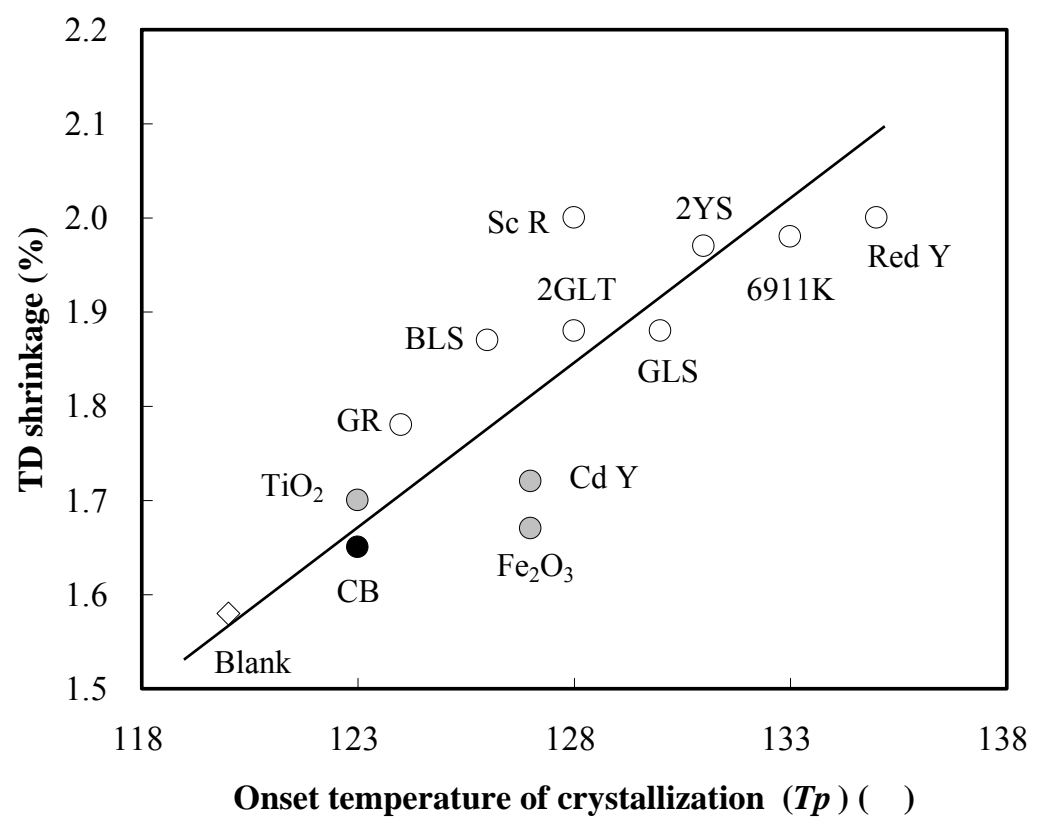

Fig. 3

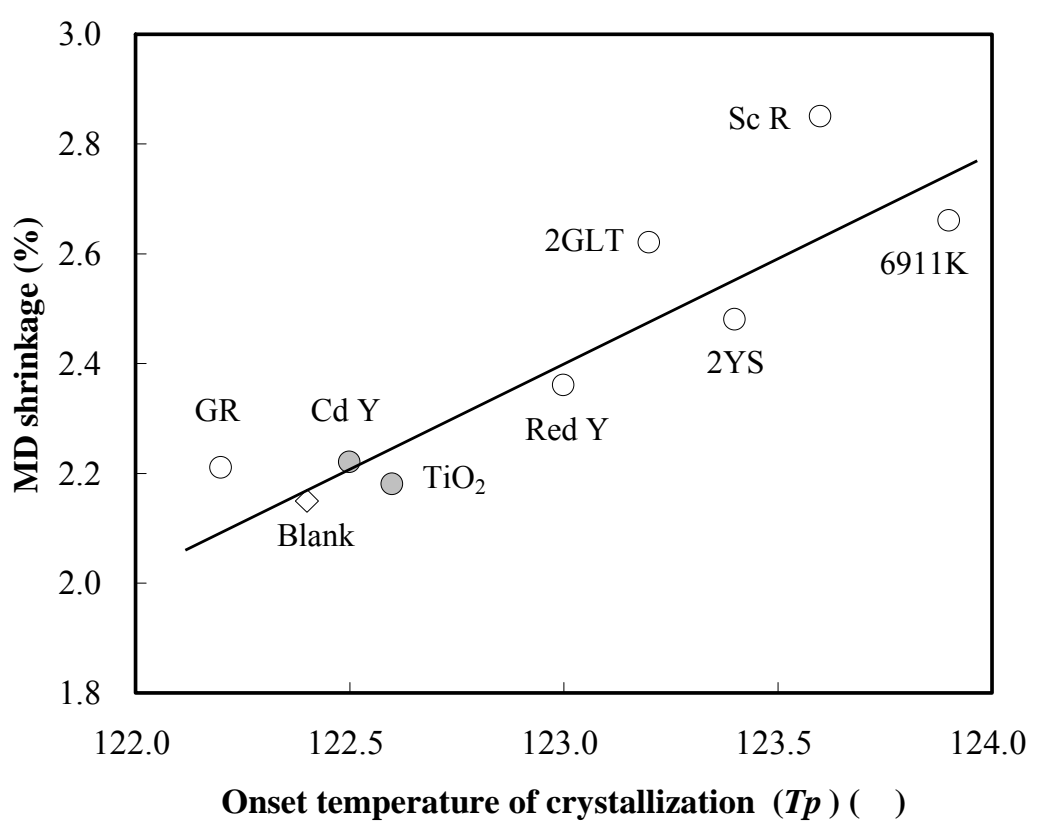

Fig. 4 


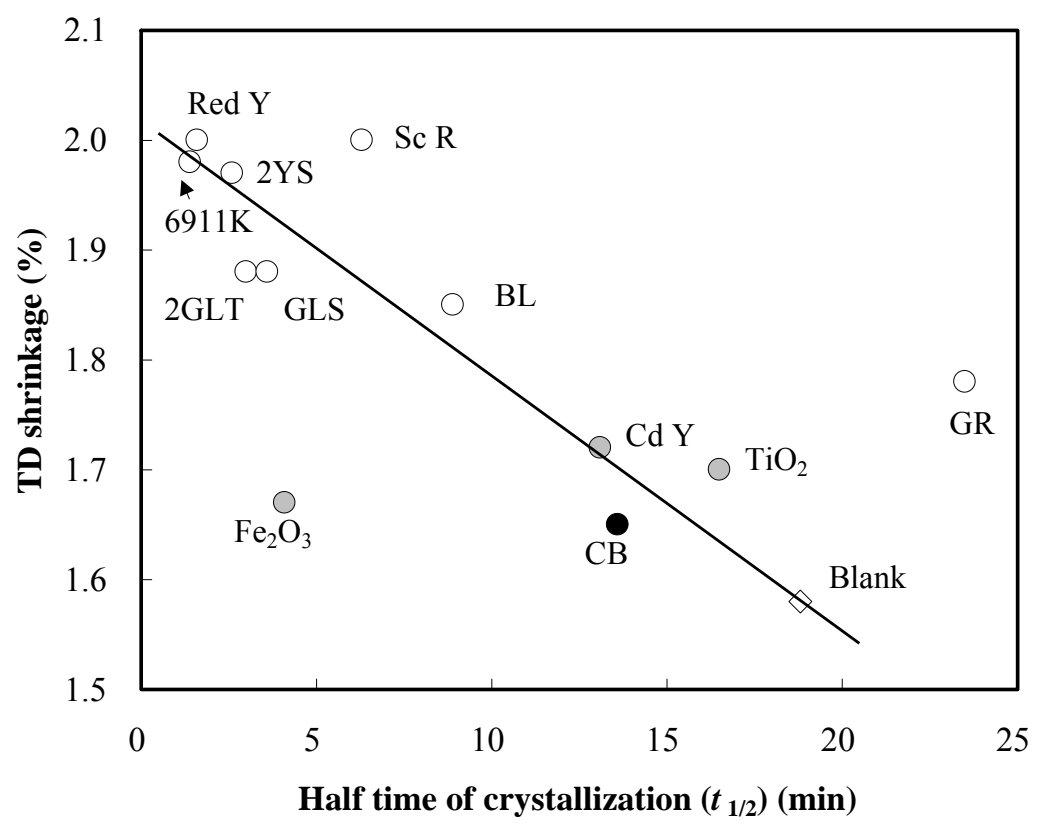

Fig. 5

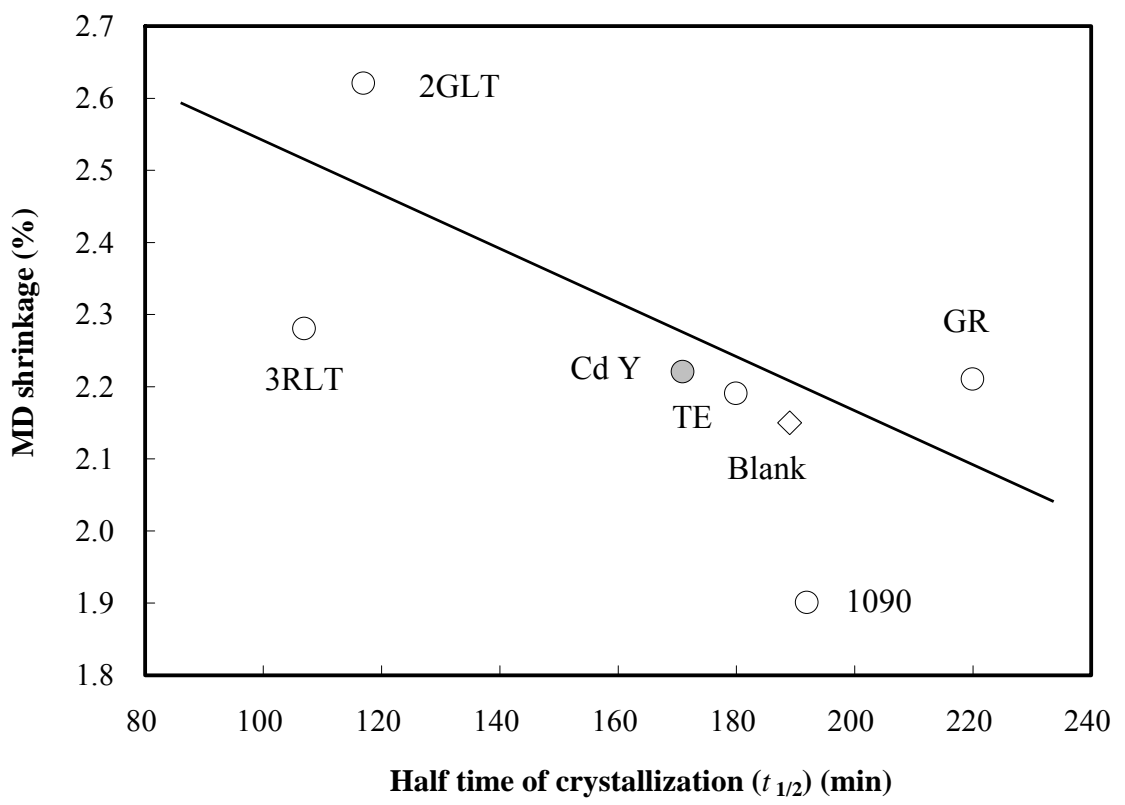

Fig. 6

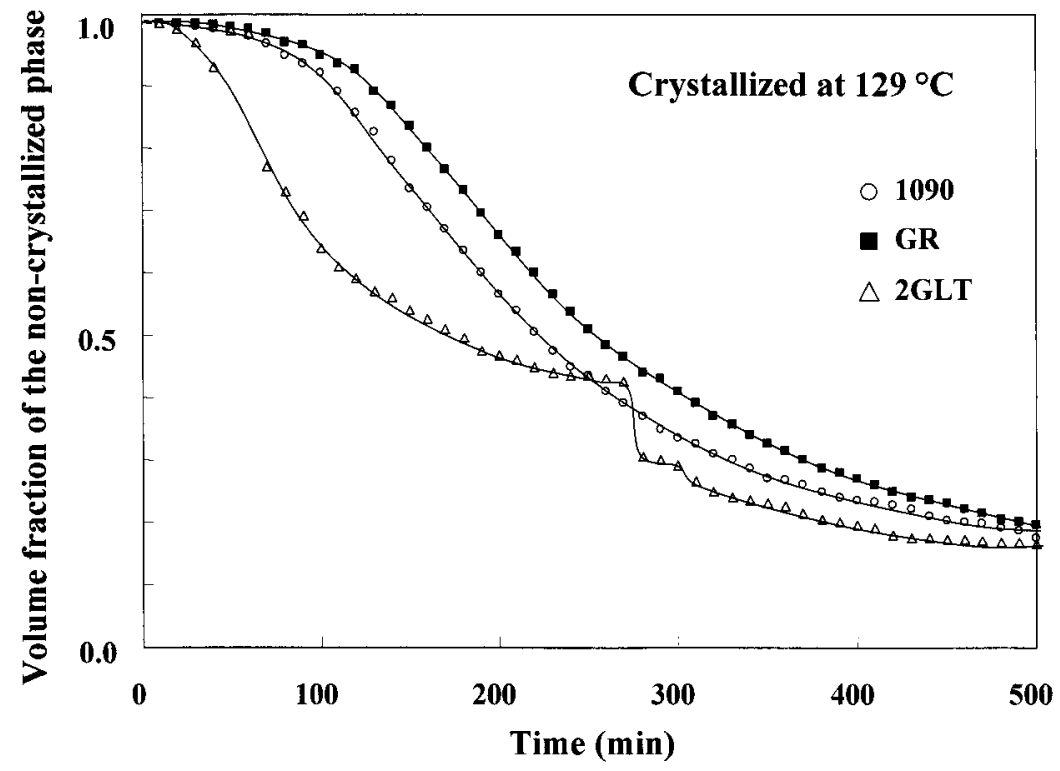

Fig. 7 